\title{
Hydro-without-Hydro Framework for Simulations of Black Hole-Neutron Star Binaries
}

\author{
Carlos F. Sopuerta, ${ }^{1}$ Ulrich Sperhake, ${ }^{1,2}$ and Pablo Laguna ${ }^{1, *}$ \\ ${ }^{1}$ Center for Gravitational Wave Physics, Penn State University, University Park, PA 16802 \\ ${ }^{2}$ Theoretisch-Physikalisches Institut, Friedrich-Schiller-Universität Jena, Max-Wien-Platz 1, 07743 Jena, Germany
}

(Dated: June 28, 2018)

\begin{abstract}
We introduce a computational framework which avoids solving explicitly hydrodynamic equations and is suitable to study the pre-merger evolution of black hole - neutron star binary systems. The essence of the method consists of constructing a neutron star model with a black hole companion and freezing the internal degrees of freedom of the neutron star during the course of the evolution of the space-time geometry. We present the main ingredients of the framework, from the formulation of the problem to the appropriate computational techniques to study these binary systems. In addition, we present numerical results of the construction of initial data sets and evolutions that demonstrate the feasibility of this approach.
\end{abstract}

PACS numbers: 04.25.Dm, 04.30.Db, 95.30.Sf, 97.80.-d

Keywords: Numerical relativity, black hole-neutron star binaries, gravitational wave sources.

\section{INTRODUCTION}

The coalescence of compact binaries are among the most important sources of gravitational waves to be detected by ground-based laser interferometers like LIGO, TAMA, GEO, and VIRGO. These observational efforts have served as the primary motivation to simulate compact binary systems. The simulations will provide crucial information in support of the data analysis. Most of the focus has been on the simulation of black hole (BH) binaries and neutron star (NS) binaries, leaving aside the BH-NS binary system (see 1, 2, 3] for recent progress). From the point of view of Numerical Relativity, the system BH-NS exhibits a dual challenge. It has the difficulties of evolving the geometry in the vicinity of BHs together with the difficulties inherent to magneto-hydrodynamical calculations.

From an astrophysical point of view, NS-BH binaries have the added interest of their potential relevance to gamma ray bursts. Observations of short gamma ray bursts (see [4, 5, 6, 7] and more recently [8, 9, 10]) suggest that the coalescence of NS binaries and/or BH-NS binaries are the underlying mechanism in the central engine for those bursts that last about $0.3 \mathrm{~s}$.

Due to the high computational cost of simulations of BH-NS binary systems (and the high cost of developing the appropriate computational infrastructure) it is desirable to have a framework in which to study certain dynamical regimes of this system, relevant to gravitational-wave physics, that admit the use of approximations and thus facilitates a drastic reduction of the computational resources required. In this sense, we are particularly interested in the stages of the evolution where the predictions of post-Newtonian methods become unreliable and where numerical relativistic simulations should take over. The hydro-without-hydro approximation that we propose in this paper aims at covering such part of the dynamical regime where the dynamical timescales related with deformations of the NS due to tidal effects are much bigger than the orbital timescales. In this regime we can freeze most of the hydrodynamical degrees of freedom and evolve a finite number of them by using appropriate approximations, focusing the attention on the radiation reaction effects in the orbit. It is this reduction of degrees of freedom which avoids the use of hydrodynamical computations. Such an approach may also be relevant for extreme-mass-ratio binary systems whose dynamics are driven by radiation-reaction effects. The main goal of this paper is then to describe the setup for this type of simulations and test, by extending an existing code for BH evolutions, the basic ingredients of this framework. These ingredients can be summarized as follows:

a. Spacetime description: We use a full numerical relativity description of the spacetime based on the $3+1$ BSSN formulation (Baumgarte, Shapiro 11], and Shibata, Nakamura 12]) of Einstein's equations as implemented in the 3D numerical relativity code MAYA (described in detail in 13, 14]). The MAYA code is written by using the computational toolkit CACTUS [15] and the Mesh Refinement package CARPET [16, 17]. The key extension of this code regarding this project is the introduction of the matter source terms associated with the NS and the associated infrastructure to evolve the matter degrees of freedom.

\footnotetext{
*Also at Institute for Gravitational Physics and Geometry, Departments of Astronomy \& Astrophysics and Physics
} 
b. Construction of Initial Data: Initial data is constructed by superposing a Schwarzschild BH in Kerr-Schild (KS) coordinates with a boosted Tolman-Oppenheimer-Volkoff (TOV) 18, 19 model in a coordinate system that has similarities with the ingoing-Eddington-Finkelstein coordinate system. The TOV model describes the equilibrium configuration of a stationary, relativistic star in spherical symmetry and thus represents a convenient description of the matter sources in the BH-NS system at hand. The superposition is carried out by using a procedure analogous to that used for the construction of binary BH initial data initial data in KS coordinates [20. Even though the resulting initial data do not satisfy the constraint equations exactly, the constraint violations are comparatively small because the individual BH and NS models do represent exact solutions of the Einstein field equations. In this work we will investigate in detail the properties of these initial data from a physical and mathematical point of view. We will also discuss potential modifications of the data with regard to their repercussions on the physical content. Finally we illustrate how adequate evolutions, even in the case of more extreme mass ratios, are facilitated by the modern infrastructure of numerical relativity.

c. Description and motion of the NS: In the hydro without hydro approximation pursued in this work, we need to address two issues relating to the description of a particular type of energy-momentum distribution: the update of the matter sources that appear in the BSSN evolution equations and the motion of such a distribution. In a full numerical relativity setup this is not necessary as the complete evolution of the matter sources is taken care of by the Einstein field- and the energy-momentum conservation equations. In reducing the degrees of freedom in the way we are proposing, one is usually left with an approximation scheme in which the internal and external motions are cleanly separated, even though they are coupled in general. The external motion consists in the motion of a reference point of the matter distribution, typically a relativistic generalization of the Newtonian concept of center of mass, whereas the internal motion consists in the evolution of the parameters describing, for instance, the deformations of the matter distributions due to tidal deformations. As a consequence of reducing the number of degrees of freedom, the description of the motion of the energy-momentum distribution in our setup consists of a set of ordinary differential equations (ODEs). The matter sources, as they appear in the evolution equations for the spacetime geometry are thus updated by merely substituting the values of the energy-momentum quantities after the motion of the matter has been determined. In this paper, we consider the simplest such description of the NS, namely that of a star rigidly moving along a prescribed trajectory. By using this simplification we ignore internal motions as well as radiation-reaction and finite size effects due to the external gravitational field. More specifically, the matter sources are prescribed by taking particular density and pressure profiles from a TOV model which are going to be maintained along the evolution. The only way in which the sources change is through the rigid bulk motion of the center of the TOV model along a fixed trajectory. The reason for considering such a simplified model is to perform a feasibility study of this method and thus lay the foundation for future studies of more realistic physical configurations of the BH-NS binary system. A similar approach to that proposed here has recently been used by Bishop et al 21] in the framework of a characteristic formulation of the Einstein equations.

The plan of this paper is as follows: In section 1 we present the mathematical description of the different ingredients of our framework: spacetime geometry and dynamics (IIA), the descriptions of the BH and the NS IIIB and IIC respectively), the initial data for the BH-NS system (IID), the dynamics of the NS (IIE). In section the computational framework and report on the results of numerical tests we have performed to analyze the initial data (subsection IIIB) and to test time evolutions with matter sources (IIIC). We conclude in section IV with a discussion of our findings. For the purpose of presenting the relativistic equations we set $c=G=1$ throughout this work.

\section{THEORETICAL FOUNDATIONS}

In this section we describe the different ingredients that constitute the mathematical framework that we are proposing for the numerical description in the time domain of BH-NS binary systems. For this purpose we need to consider both, the generation of initial data, and the evolution of these data. In the construction of initial data we closely follow a procedure used for a binary BH systems: the superposition of two BH solutions in KS form (cf. [20, 22, 23, 24]). This technique is based on the properties of the KS form of a single $\mathrm{BH}$ metric and its invariance under Lorentz transformations of the coordinates. Then, starting from two single BHs in KS coordinates (and different coordinate origin) a Lorentz boost is applied to each of them and then, by identifying the coordinate systems, a superposed metric is constructed. This metric is no longer an exact solution of the Einstein field equations (the constraint are not satisfied), but it is an approximation which improves as the $\mathrm{BH}$ separation increases. This type of data has been shown to work well in numerical relativity simulations (see, e.g. [24]). It has the advantage that one can consider Kerr $\mathrm{BH}$ initial data without the need to worry about spurious radiation that may originate in initial data constructions based on conformally-flat slicings.

The evolution of the data is performed in analogy to that of BH-data, with one exception: the addition of matter 
source terms on the right hand side of the evolution equations. We thus obtain a fully non-linear evolution of the geometry of the spacetime. In contrast, we substantially reduce the degrees of freedom of the matter sources. We will discuss in detail in this section, how this enables us to obtain an approximate evolution of the matter data.

\section{A. Description of the Spacetime Geometry}

We begin our description of the mathematical model with the spacetime geometry. In this work we follow the standard 3+1 splitting of Einstein's equations based on the Arnowitt-Deser-Misner (ADM) formulation [25] (see [26, 27 for details). There it is assumed that the spacetime has topology $\mathbb{R} \times \Sigma$, and hence can be foliated by the level surfaces $\Sigma(t)$ of a function $t\left(x^{\mu}\right)$. The unit normal to the hypersurfaces $\Sigma(t), n^{\mu}=-\alpha \nabla^{\mu} t$, satisfies $g_{\mu \nu} n^{\mu} n^{\nu}=-1$. It can be used to decompose the four-metric according to

$$
\mathrm{g}_{\mu \nu}=h_{\mu \nu}-n_{\mu} n_{\nu}
$$

where $h^{\mu}{ }_{\nu}$ is the projector orthogonal to $n^{\mu}\left(h^{\mu}{ }_{\nu} n^{\nu}=0\right)$. A vector field $t^{\mu}$ threading the spacetime is introduced, $t^{\mu} \nabla_{\mu} t=1$, in terms of which we can introduce the lapse function and shift vector through the relations $\alpha=-t^{\mu} n_{\mu}$ and $\beta^{\mu}=t^{\mu}-\alpha n^{\mu}$ respectively. Then, using a system of spatial coordinates $\left\{x^{i}\right\}$ adapted to the hypersurfaces $\Sigma(t)$, the metric takes the well-known form:

$$
d s^{2}=-\alpha^{2} d t^{2}+\gamma_{i j}\left(d x^{i}+\beta^{i} d t\right)\left(d x^{j}+\beta^{j} d t\right)
$$

where $\gamma_{i j}=h_{i j}$ are the components of the spatial metric of the hypersurfaces $\Sigma(t)$. The extrinsic curvature of $\Sigma(t)$ is defined as

$$
K_{\mu \nu}=-\frac{1}{2} £_{n} h_{\mu \nu}
$$

where $£$ denotes the Lie derivative operator. We thus obtain

$$
\hat{\partial}_{t} \gamma_{i j}=-2 \alpha K_{i j}
$$

where we have introduced the time derivative operator:

$$
\hat{\partial}_{t}=\partial_{t}-£_{\beta}
$$

Because we are dealing with a physical system that contains matter, we also need to apply the $3+1$ splitting to the energy-momentum tensor $T^{\mu \nu}$. This can be done by decomposing $T^{\mu \nu}$ with respect to the unit normal $n_{\mu}$ :

$$
T^{\mu \nu}=E n^{\mu} n^{\nu}+P h^{\mu \nu}+2 J^{(\mu} n^{\nu)}+\Pi^{\mu \nu}
$$

where the different quantities that appear in this expression are defined as follows:

$$
\begin{aligned}
E & =T^{\mu \nu} n_{\mu} n_{\nu}, \\
P & =\frac{1}{3} h_{\mu \nu} T^{\mu \nu}, \\
J^{\mu} & =-h^{\mu}{ }_{\nu} T^{\nu \tau} n_{\tau}, \\
\Pi_{\mu \nu} & =h_{\mu \tau} h_{\nu \sigma} T^{\tau \sigma}-P h_{\mu \nu} .
\end{aligned}
$$

Applying the $3+1$ splitting to Einstein's equations we obtain the Evolution equations:

$$
\begin{aligned}
\hat{\partial}_{t} \gamma_{i j} & =-2 \alpha K_{i j}, \\
\hat{\partial}_{t} K_{i j} & =-D_{i} D_{j} \alpha+\alpha\left[R_{i j}+K K_{i j}-2 K_{i k} K_{j}^{k}-\Pi_{i j}-\frac{1}{2}(E-P) \gamma_{i j}\right],
\end{aligned}
$$

and the Constraint equations:

$$
\begin{aligned}
\mathcal{H} & \equiv R+K^{2}-K_{i j} K^{i j}-2 E=0, \\
\mathcal{P}^{i} & \equiv D_{j}\left(K^{i j}-\gamma^{i j} K\right)-J^{i}=0 .
\end{aligned}
$$

Here, $D_{i}$ is the covariant derivative associated with the 3-metric $\gamma_{i j}, R_{i j}$ is the three-dimensional Ricci tensor, $R=\gamma^{i j} R_{i j}$ the Ricci scalar, and $K$ is the trace of $K_{i j}$. Equations (1112) and (13 14) constitute the basic 3+1 setup 
and are known as the ADM equations 25]. We are going to use a modification of this formulation due to Shibata and Nakamura [12], and Baumgarte and Shapiro [11], now known as the BSSN 3+1 formulation of Einstein's equations, which has been found to result in vastly improved stability properties in numerical simulations compared with the ADM equations. The way in which the BSSN formulation is introduced from the ADM formulation is as follows: We introduce new variables based on a trace decomposition of the extrinsic curvature and a conformal rescaling of both the metric and the extrinsic curvature. The trace-free part $A_{i j}$ of the extrinsic curvature is defined by

$$
A_{i j}=K_{i j}-\frac{1}{3} \gamma_{i j} K
$$

Next, one introduces a conformal metric $\tilde{\gamma}_{i j}$ in terms of the physical metric by

$$
\gamma_{i j}=\psi^{4} \tilde{\gamma}_{i j}
$$

The value of the conformal factor $\psi$ can be fixed by demanding the determinant of the conformal metric $\tilde{\gamma}_{i j}$ to be unity:

$$
\psi=\gamma^{1 / 12}, \quad \tilde{\gamma}_{i j}=\psi^{-4} \gamma_{i j}=\gamma^{-1 / 3} \gamma_{i j}, \quad \tilde{\gamma}=1
$$

where $\gamma$ and $\tilde{\gamma}$ are the determinants of $\gamma_{i j}$ and $\tilde{\gamma}_{i j}$ respectively. Then, instead of $\gamma_{i j}$ and $K_{i j}$ we can use the variables

$$
\begin{aligned}
\phi & =\ln \psi=\frac{1}{12} \ln \gamma, \\
K & =\gamma_{i j} K^{i j}, \\
\tilde{\gamma}_{i j} & =e^{-4 \phi} \gamma_{i j}, \\
\tilde{A}_{i j} & =e^{-4 \phi} A_{i j},
\end{aligned}
$$

where $\tilde{\gamma}_{i j}$ has determinant 1 and $\tilde{A}_{i j}$ has vanishing trace. Moreover, we also introduce the following conformal connection functions

$$
\tilde{\Gamma}^{i}=\tilde{\gamma}^{j k} \tilde{\Gamma}_{j k}^{i}=-\partial_{j} \tilde{\gamma}^{i j}
$$

where $\tilde{\Gamma}_{j k}^{i}$ denotes the Christoffel symbols associated with the conformal metric. To obtain the second equality we have to use the fact that the determinant of the conformal spatial metric, $\tilde{\gamma}$, is unity.

The variables used in the BSSN formulation are: $\phi, K, \tilde{\gamma}_{i j}, \tilde{A}_{i j}$, and $\tilde{\Gamma}^{i}$. The set of evolution equations for these variables that we are going to use, and which can be derived from the previous relations, is given by

$$
\begin{aligned}
\hat{\partial}_{t} \tilde{\gamma}_{i j} & =-2 \alpha \tilde{A}_{i j} \\
\hat{\partial}_{t} \phi & =-\frac{1}{6} \alpha K \\
\hat{\partial}_{t} \tilde{A}_{i j} & =e^{-4 \phi}\left[-\left(D_{i} D_{j} \alpha\right)^{T F}+\alpha\left(R_{i j}^{T F}-8 \pi \Pi_{i j}\right)\right]+\alpha\left(K \tilde{A}_{i j}-2 \tilde{A}_{i k} \tilde{A}_{j}^{k}\right) \\
\hat{\partial}_{t} K & =-D^{i} D_{i} \alpha+\alpha\left[\tilde{A}_{i j} \tilde{A}^{i j}+\frac{1}{3} K^{2}+4 \pi(E+3 P)\right] \\
\partial_{t} \tilde{\Gamma}^{i} & =\beta^{j} \partial_{j} \tilde{\Gamma}^{i}-\tilde{\Gamma}^{j} \partial_{j} \beta^{i}-2 \tilde{A}^{i j} \partial_{j} \alpha+2 \alpha\left(\tilde{\Gamma}_{j k}^{i} \tilde{A}^{j k}+6 \tilde{A}^{i j} \partial_{j} \phi-\frac{2}{3} \tilde{\gamma}^{i j} \partial_{j} K-8 \pi e^{4 \phi} J^{i}\right) \\
& +\tilde{\gamma}^{j k} \partial_{j} \partial_{k} \beta^{i}+\frac{1}{3} \tilde{\gamma}^{i j} \partial_{j} \partial_{k} \beta^{k}+\frac{2}{3} \tilde{\Gamma}^{i} \partial_{j} \beta^{j}+\left(\chi+\frac{2}{3}\right)\left(\tilde{\Gamma}^{i}-\tilde{\gamma}^{j k} \tilde{\Gamma}_{j k}^{i}\right) \partial_{j} \beta^{j} .
\end{aligned}
$$

Here the superscript $T F$ denotes the trace-free part (with respect to the metric $\gamma_{i j}$ ). In this context, it is important to note that $\phi$ is defined in terms of the determinant of the 3 -metric $\gamma_{i j}$, which is a scalar density of weight 2 , and that $\tilde{\gamma}_{i j}$ and $\tilde{A}_{i j}$ are tensor densities of weight $-2 / 3$. Finally, $\chi$ is a free parameter [it multiplies a quantity that vanishes at the analytic level by virtue of the definition of the quantities $\left.\tilde{\Gamma}^{i}(22)\right]$ that has been set to $2 / 3$ for all simulations reported here. This value has been suggested by numerical experiments.

\section{B. BH description: Kerr-Schild form of a Schwarzschild BH}

Although spinning BHs are likely to be more interesting from an astrophysical point of view, especially in the case of extreme-mass-ratio binaries, in this work we restrict our attention to non-rotating Schwarzschild BHs. We do 
note, however, that for the case of KS coordinates, the fundamental properties of spinning and non-spinning black holes are the same from a numerical point of view (see, for example, the successful evolutions of single Kerr BHs obtained in 28] ). This feature represents a key motivation in our choice of coordinates and is not obviously satisfied by coordinates which are not adapted to the spinning cases (for instance, all the coordinate systems that assume an initially conformally-flat spatial geometry).

In this section we describe the contribution to the initial data of the $\mathrm{BH}$, that is we need to prescribe initial data for the functions $\left(\phi, K, \tilde{\gamma}_{i j}, \tilde{A}_{i j}, \tilde{\Gamma}^{i}\right)$. These arise from the KS form of the metric, which can be conveniently written as the sum of a flat space metric plus a term that factorizes as the product of a light-like vector with itself according to

$$
d s^{2}=\left(\eta_{\mu \nu}+2 H \ell_{\mu} \ell_{\nu}\right) d x^{\mu} d x^{\nu} .
$$

Here $\eta_{\mu \nu}=\operatorname{diag}(-1,1,1,1)$ is the Minkowski metric in Cartesian coordinates $\left\{t, x^{i}\right\}$ and $\ell^{\mu}$ is a null vector field

$$
\ell_{\mu} d x^{\mu}=-d t-\frac{x_{i}}{r} d x^{i}, \quad r^{2}=\delta_{i j} x^{i} x^{j},
$$

where $x_{i}=x^{i}$. This light-like vector field has been chosen so that it corresponds to ingoing light rays. Finally, $H$ is a scalar given by

$$
H=\frac{M_{\mathrm{BH}}}{r},
$$

where $M_{\mathrm{BH}}$ is the $\mathrm{BH}$ mass.

The metric quantities are then independent of the time $t$ which slices the spacetime

$$
\begin{gathered}
\alpha_{\mathrm{BH}}^{2}=\frac{1}{1+2 H}, \quad \beta_{\mathrm{BH}}^{i}=\frac{2 H}{1+2 H} \ell^{i}, \\
\gamma_{i j}^{\mathrm{BH}}=\delta_{i j}+2 H \ell_{i} \ell_{j}, \quad \gamma_{\mathrm{BH}}^{i j}=\delta^{i j}-\frac{2 H}{1+2 H} \ell^{i} \ell^{j}, \\
\gamma_{\mathrm{BH}}=\operatorname{det}\left(\gamma_{i j}^{\mathrm{BH}}\right)=1+2 H=\alpha_{\mathrm{BH}}^{-2} .
\end{gathered}
$$

\section{NS matter description: The Tolman-Oppenheimer-Volkoff stellar model}

The main approximation in our description of the BH-NS binary system lies in the description of the NS. The main goal of this approximation is to provide a framework in which one avoids solving the hydrodynamical equations governing the NS matter fields while at the same time being able to have a reasonable approximation of the main dynamical aspects of the evolution of such a system. The implementation of this idea requires two ingredients: (i) The prescription of the profiles for the different matter variables and the construction of initial data for the geometry. (ii) The evolution of the matter profiles. Regarding the second point, in this paper we take the simplest description, namely, to consider rigid profiles that follow a prescribed trajectory. In subsection ஹE we briefly discuss how this description can be refined in future work to obtain physically more realistic models.

With regard to the first point, the matter profiles for the NS matter variables are obtained by using an exact solution representing an isolated NS star, more precisely a TOV model. With regard to the eventual superposition with the BH, we will apply two modifications to the standard TOV metric: i) a transformation to KS-like coordinates, and ii) apply a boost transformation to account for the motion of the star relative to the black hole. Our starting point is the TOV metric in Schwarzschild coordinates

$$
\mathrm{g}_{\alpha \beta} d x^{\alpha} d x^{\beta}=-A^{2}(\bar{R}) d \bar{T}^{2}+B^{2}(\bar{R}) d \bar{R}^{2}+\bar{R}^{2} d \Omega^{2},
$$

where $d \Omega^{2}=d \theta^{2}+\sin ^{2} \theta d \varphi^{2}$. The energy momentum tensor of a TOV star is that of a perfect fluid and is given by

$$
T_{\alpha \beta}=(\rho+p) u_{\alpha} u_{\beta}+p \mathrm{~g}_{\alpha \beta},
$$

where $u_{\alpha}=(-A, 0,0,0)$ is the 4 -velocity of the fluid elements. It is convenient to introduce the mass function $m(\bar{R})$ by

$$
B^{-2}=1-2 m / \bar{R} .
$$


Then, the TOV equations are:

$$
\begin{aligned}
\frac{A^{\prime}}{A} & =\frac{m+4 \pi p \bar{R}^{3}}{\bar{R}(\bar{R}-2 m)}=-\frac{p^{\prime}}{\rho+p}, \\
m^{\prime} & =4 \pi \rho \bar{R}^{2}
\end{aligned}
$$

where a prime denotes differentiation with respect to $\bar{R}$. We are going to consider the case of a barotropic Equation of State (EoS), specifically a polytropic one:

$$
p=k \rho^{\Gamma}, \quad \Gamma=1+\frac{1}{n},
$$

where the polytropic coefficient $k$ and the polytropic index $n$ are constants. Equations (3738) determine a oneparameter family of TOV solutions, which can be parametrized by the value of the central density $\rho_{\mathrm{c}}=\rho(\bar{R}=0)$. The sound speed of the matter distribution is given by $c_{s}^{2}=d p / d \rho$.

Bearing in mind the eventual superposition of the BH and NS metrics in analogy to the construction of KS binary $\mathrm{BH}$ data, we are going to apply coordinate transformations to the TOV solution that resemble the coordinate transformations used in the $\mathrm{BH}$ case. In the case of $\mathrm{BHs}$, the first coordinate transformation aims at changing the time coordinate so that it is adapted to light-like geodesics, typically ingoing ones. For a Schwarzschild $\mathrm{BH}$, this corresponds to the KS coordinate system. In the case of a NS we follow the same idea. A straightforward calculation shows that after applying the coordinate transformation

$$
d \hat{T}=d \bar{T}+(B / A-1) d \bar{R}, \quad d R=d \bar{R} .
$$

the ingoing radial light-like geodesics are given by $\hat{T}+R$. This transformation, however, is not suitable for a three-dimensional numerical treatment because it leads to a coordinate singularity at the centre of the star when we transform to Cartesian coordinates [by using the transformation given below in equation (44)]. It is possible, though, to find a coordinate transformation analogous to (40) that satisfies all regularity requirements at the stellar centre. For this purpose we define the new coordinate time $T$ by

$$
d T=d \bar{T}+\left(\frac{B}{A}-\frac{1}{A B}\right) d \bar{R}
$$

The resulting line element is then given by:

$$
d s^{2}=-A^{2} d T^{2}+2 A B\left(1-B^{-2}\right) d T d R+\left(2-B^{-2}\right) d R^{2}+R^{2} d \Omega^{2} .
$$

By virtue of Birkhoff's theorem, the geometry exterior to the star is necessarily described by the Schwarzschild metric. In order to combine the interior and exterior metric, we need to match them in a smooth way at the stellar surface. It is a remarkable property of the interior metric (42) that it still smoothly matches to the exterior (KS) solution and thus leads to coordinates adapted to the ingoing null geodesic structure outside the star. This follows straightforwardly from performing the substitution $A^{2}=B^{-2}=1-2 M_{\mathrm{NS}} / R$, where $M_{\mathrm{NS}}$ is the NS mass. In consequence, the interior and exterior geometries match smoothly (in the sense of the Lichnerowicz junction conditions [29, 30]), at a hypersurface $R=R_{\mathrm{NS}}=$ constant, provided the TOV model satisfies the following conditions

$$
\left.A^{2}\right|_{R=R_{\mathrm{NS}}}=1-2 M_{\mathrm{NS}} / R_{\mathrm{NS}}, \quad p\left(R_{\mathrm{NS}}\right)=0,
$$

which have the obvious consequences: $m\left(R_{\mathrm{NS}}\right)=M_{\mathrm{NS}}$ and $\left.B^{-2}\right|_{R=R_{\mathrm{NS}}}=1-2 M_{\mathrm{NS}} / R_{\mathrm{NS}}$ (or equivalently $\left.A B\right|_{R=R_{\mathrm{NS}}}=$ 1 ), and clearly $R_{\mathrm{NS}}$ represents the NS surface.

Next, we apply the coordinate change from spherical $(T, R, \theta, \varphi)$ to the Cartesian-like coordinates $\left(T, X^{i}\right)$. These coordinates are related by

$$
\left(X^{i}\right)=(X, Y, Z)=(R \sin \theta \cos \varphi, R \sin \theta \sin \varphi, R \cos \theta) .
$$

The resulting line element is

$$
d s^{2}=-A^{2} d T^{2}+2 A B\left(1-B^{-2}\right) d T \frac{X_{i}}{R} d X^{i}+\left[\delta_{i j}+\left(1-B^{-2}\right) \frac{X_{i} X_{j}}{R^{2}}\right] d X^{i} d X^{j},
$$

where $R$ is considered a function of $X^{i}$ given by $R^{2}=\delta_{i j} X^{i} X^{j}$, and $X_{i}=X^{i}$. At this point, we can see that $B \rightarrow 1$ as $R \rightarrow 0$, i. e. the metric is regular at the origin $X^{i}=0$ of the stellar object. This would not have been the case for the coordinate transformation (40) which would have lead to a cusp at the origin of the star. 
The ADM metric variables associated with the slicing $\{T=$ constant $\}$ are:

$$
\begin{aligned}
\alpha_{\mathrm{C}}^{2} & =\frac{A^{2} B^{2}}{2-B^{-2}}, \\
\beta_{\mathrm{C}}^{i} & =A B \frac{1-B^{-2}}{2-B^{-2}} \frac{X^{i}}{R}, \\
\gamma_{i j}^{\mathrm{C}} & =\delta_{i j}+\left(1-B^{-2}\right) \frac{X_{i} X_{j}}{R^{2}},
\end{aligned}
$$

where we have used the tag ' $\mathrm{C}$ ' to distinguish the quantities associated with this particular slicing. We now address the next step in the construction of the superposed geometry, the Lorentz boost. We first note, that the NS metric (45) cannot be cast in the KS form (28), which is preserved under Lorentz transformations. While the NS metric can still be written in the form $\eta_{\mu \nu}+H_{\mu \nu}$, the resulting $H_{\mu \nu}$ cannot be factorized in terms of a null vector field, where $H_{\mu \nu} \rightarrow 0$ as $R \rightarrow \infty$. On the other hand, the form $\eta_{\mu \nu}+H_{\mu \nu}$ is still Lorentz invariant. We thus apply a coordinate change from the Cartesian-like coordinates $\left(T, X^{i}\right)$ of Eq. (45) to a different set of Cartesian-like coordinates $\left(t, x^{i}\right)$ via a Lorentz boost. These two sets of coordinates are related by the following expressions:

$$
\begin{aligned}
T & =\gamma\left[t-\delta_{i j} v^{i}\left(x^{j}-z^{j}\right)\right], \\
X^{i} & =-\gamma t v^{i}+\left[\delta_{j}^{i}+(\gamma-1) \frac{v^{i} v_{j}}{v^{2}}\right]\left(x^{j}-z^{j}\right),
\end{aligned}
$$

where $v^{i}$ are the components of the boost velocity $\left(v_{i}=v^{i}\right), v^{2}$ is $\delta_{i j} v^{i} v^{j}, \gamma$ is $\left(1-v^{2}\right)^{-1 / 2}$, and $z^{i}$ are the spatial components of the compact object's location. In the course of the evolution, the position vector $z^{i}$ needs to be evolved by solving the equations governing the external dynamics of the NS. In this paper it is going to be a prescribed trajectory.

After carrying out the transformation (49) the ADM metric variables associated with the slicing $\{t=$ constant $\}$ can be written as

$$
\begin{aligned}
\alpha_{\mathrm{NS}}^{2} & =\alpha_{\mathrm{C}}^{2} \gamma^{-2}\left[\left(1-v_{i} \beta_{\mathrm{C}}^{i}\right)^{2}-\alpha_{\mathrm{C}}^{2} \gamma_{\mathrm{C}}^{i j} v_{i} v_{j}\right]^{-1} \\
\beta_{\mathrm{NS}}^{i} & =-\frac{\gamma}{1+\gamma} v^{i}+\gamma \alpha_{\mathrm{NS}}^{2}\left[\alpha_{\mathrm{C}}^{-2}\left(1-v_{i} \beta_{\mathrm{C}}^{i}\right)\left(\beta_{\mathrm{C}}^{i}-\frac{\gamma}{1+\gamma} v^{i}\right)+\gamma_{\mathrm{C}}^{i j} v_{j}\right] \\
\gamma_{i j}^{\mathrm{NS}} & =\gamma_{i j}^{\mathrm{C}}-2 \gamma v_{(i} \gamma_{j) k}^{\mathrm{C}}\left(\beta_{\mathrm{C}}^{k}-\frac{\gamma}{1+\gamma} v^{k}\right)+\gamma^{2}\left[\gamma_{k l}^{\mathrm{C}}\left(\beta_{\mathrm{C}}^{k}-\frac{\gamma}{1+\gamma} v^{k}\right)\left(\beta_{\mathrm{C}}^{l}-\frac{\gamma}{1+\gamma} v^{l}\right)-\alpha_{\mathrm{C}}^{2}\right] v_{i} v_{j}
\end{aligned}
$$

where $\alpha_{\mathrm{C}}, \beta_{\mathrm{C}}^{i}$, and $\gamma_{i j}^{\mathrm{C}}$ are given in equations (46)-(48) in terms of the coordinates $\left(T, X^{i}\right)$. With expressions (51)-(53) we complete the geometric description of the NS. They will be used below to construct the initial data by superposing the $\mathrm{BH}$ and the NS solutions.

We denote the matter source terms associated with the foliation $\{t=$ const. $\}$, by $\left(E_{\mathrm{NS}}, P_{\mathrm{NS}}, J_{\mathrm{NS}}^{i}, \Pi_{i j}^{\mathrm{NS}}\right)$. These can be obtained from the expressions (7)-(10), bearing in mind that the normal to this foliation is $n_{\mu} d x^{\mu}=-\alpha_{\mathrm{NS}} d t$ and the components of the fluid four-velocity are

$$
u^{t}=\gamma u^{T}, \quad u^{i}=u^{t} v^{i}
$$

where $u^{T}=A^{-1}$. Then, the result is

$$
\begin{aligned}
E_{\mathrm{NS}} & =(\rho+p)\left(\frac{\alpha_{\mathrm{NS}} \gamma}{A}\right)^{2}-p \\
P_{\mathrm{NS}} & =\frac{1}{3}\left[(\rho+p)\left(\frac{\alpha_{\mathrm{NS}} \gamma}{A}\right)^{2}-\rho+2 p\right] \\
J_{\mathrm{NS}}^{i} & =\alpha_{\mathrm{NS}} \frac{\gamma^{2}}{A^{2}}(\rho+p)\left(\beta_{\mathrm{NS}}^{i}+v^{i}\right) \\
\Pi_{i j}^{\mathrm{NS}} & =(\rho+p) \frac{\gamma^{2}}{A^{2}}\left(\gamma_{i k}^{\mathrm{NS}} \gamma_{j l}^{\mathrm{NS}}-\frac{1}{3} \gamma_{i j}^{\mathrm{NS}} \gamma_{k l}^{\mathrm{NS}}\right)\left(\beta_{\mathrm{NS}}^{k}+v^{k}\right)\left(\beta_{\mathrm{NS}}^{l}+v^{l}\right),
\end{aligned}
$$

where $\alpha_{\mathrm{NS}}, \beta_{\mathrm{NS}}^{i}$, and $\gamma_{i j}^{\mathrm{NS}}$ are given by (51)-(53), and $\rho$ and $p$ are the TOV energy density and isotropic pressure. 


\section{Superposition of the BH and NS: Initial Data and BSSN Source Terms}

We now have all the necessary ingredients to superpose the BH and NS spacetimes given above. Because of the presence of matter terms, this process involves two steps: (i) the construction of the geometric quantities associated with the BH-NS system and, (ii) the construction of the matter source terms associated with that geometry.

First, we superpose the geometries in complete analogy with the BH binary case (cf. 24, 31]). Let us consider, for this purpose, the BH metric given by expressions (28)-(30) and the NS metric given by expressions (51)-(53). By identifying the two spacetimes' coordinate systems, the spatial metric and extrinsic curvature of the superposition are given by

$$
\begin{aligned}
\gamma_{i j} & =\gamma_{i j}^{\mathrm{BH}}+\gamma_{i j}^{\mathrm{NS}}-\delta_{i j}, \\
K_{j}^{i} & =K_{\mathrm{BH} j}^{i}+K_{\mathrm{NS} j}^{i} .
\end{aligned}
$$

Moreover, the lapse and shift associated with the superposition are given by the following expressions:

$$
\begin{aligned}
\alpha & =\left(\alpha_{\mathrm{BH}}^{-2}+\alpha_{\mathrm{NS}}^{-2}-1\right)^{-1 / 2}, \\
\beta^{i} & =\gamma^{i j}\left(\beta_{j}^{\mathrm{BH}}+\beta_{j}^{\mathrm{NS}}\right) .
\end{aligned}
$$

Second, we need to obtain from the NS description the expressions for the matter sources that appear on the righthand sides of the BSSN equations (23)-(28). To that end, we consider the following form of the energy-momentum tensor

$$
T^{\mu \nu}=(\rho+p) U^{\mu} U^{\nu}+p \mathrm{~g}^{\mu \nu},
$$

where $\mathrm{g}^{\mu \nu}$ is the inverse of the global metric given in equations (59)-(62), $\rho$ and $p$ are the TOV energy density and isotropic pressure written in the coordinates $\left(t, x^{i}\right)$ of equations (4950), and $U^{\mu}=U^{t}\left(1, v^{i}\right)$ is the fluid velocity. The time component $U^{t}$ of the fluid velocity is determined in terms of the boost velocity $v^{i}$ and the global geometry by imposing the normalization condition $g_{\alpha \beta} U^{\alpha} U^{\beta}=-1$. The resulting expression is

$$
\frac{1}{\left(U^{t}\right)^{2}}=\alpha^{2}-\gamma_{k l}\left(\beta^{k}+v^{k}\right)\left(\beta^{l}+v^{l}\right)
$$

Following the same procedure as above, we can compute the matter quantities $\left(E, P, J^{i}, \Pi_{i j}\right)$ associated with the NS in the superposed BH-NS spacetime. We note that the normal to the foliation is now $n_{\mu} d x^{\mu}=-\alpha d t$. We thus obtain:

$$
\begin{aligned}
E & =(\rho+p)\left(\alpha U^{t}\right)^{2}-p, \\
P & =p+\frac{1}{3}(\rho+p)\left(U^{t}\right)^{2} \gamma_{i j}\left(\beta^{i}+v^{i}\right)\left(\beta^{j}+v^{j}\right)=p-\frac{1}{3}(E-\rho), \\
J^{i} & =(\rho+p) \alpha\left(U^{t}\right)^{2}\left(\beta^{i}+v^{i}\right), \\
\Pi_{i j} & =(\rho+p)\left(U^{t}\right)^{2}\left(\gamma_{i k} \gamma_{j l}-\frac{1}{3} \gamma_{i j} \gamma_{k l}\right)\left(\beta^{k}+v^{k}\right)\left(\beta^{l}+v^{l}\right),
\end{aligned}
$$

where $\alpha, \beta^{i}$, and $\gamma_{i j}$ are given by (61), (62), and (59) respectively, and $\rho$ and $p$ are the TOV energy density and isotropic pressure. These expressions are very close in form to the expressions we obtained for a single NS, the main difference being that the geometric objects now refer to the superposed spacetime instead of the TOV spacetime.

It is clear that these initial data only satisfy the constraints in the limit of infinite BH-NS separation. The constraint violations inherent to this construction, however, are expected to be small. If, for example, one considers this type of superposed data as the background metric fields for the conformal transverse-traceless method of Lichnerowicz, York and others $32,33,34,35,36]$, the conformal factor obtained from solving the Hamiltonian constraint is close to unity throughout the whole spacetime [23]. Furthermore the quality of the data can always be enhanced by increasing the distance between the BHs. Finally, this data is believed to avoid spurious radiation contamination, and in contrast to conformally-flat data, it is possible to introduce Kerr BH solutions in a straightforward way.

Bonning et al 23. have further shown that the superposed binary BH initial data lead to the correct (Newtonian) binding energy in the Newtonian limit. We can ask the same question about the prescription we have presented for BH-NS binaries. The answer is negative, and, in essence, the reason is that the way in which the NS has been introduced does not account for the deformations caused by the $\mathrm{BH}$ gravitational field. In the case of $\mathrm{BH}$ binaries the binding energy is defined as $E_{b}=E_{\mathrm{ADM}}^{\text {total }}-E_{\mathrm{BH}}^{1}-E_{\mathrm{BH}}^{2}$, where the total ADM energy is $M_{\mathrm{BH}}^{1}+M_{\mathrm{BH}}^{2}$ and the individual energies are defined in terms of the apparent horizon masses: $E_{\mathrm{BH}}^{I}=M_{\mathrm{AH}}^{I}(I=1,2)$. In the Newtonian 
limit $\left(G M /\left(c^{2} \ell\right) \ll 1\right.$, where $\ell$ denotes the coordinate separation) they are given by $[23]: E_{\mathrm{BH}}^{1} \approx M_{\mathrm{BH}}^{1}\left[1+M_{\mathrm{BH}}^{2} /(2 \ell)\right]$ and $E_{\mathrm{BH}}^{2} \approx M_{\mathrm{BH}}^{2}\left[1+M_{\mathrm{BH}}^{1} /(2 \ell)\right]$. We thus get the familiar expression $E_{b} \approx-M_{\mathrm{BH}}^{1} M_{\mathrm{BH}}^{2} / \ell$. If one of the objects is a $\mathrm{NS}$, all that changes, is the individual energy $E_{\mathrm{NS}}$ associated with the star. It is now obtained from the integration of the NS energy density over the initial slice. Including the contribution of the BH to the volume element, the result is $E_{\mathrm{NS}} \approx M_{\mathrm{NS}}\left(1+M_{\mathrm{BH}} / \ell\right)-E_{\mathrm{NS}}^{\text {self }}$, where $E_{\mathrm{NS}}^{\text {self }}$ denotes the NS self-binding energy. Therefore, we do not recover the Newtonian expression. The reason for this lies in the way in which the NS has been introduced in the superposition and the fact that the definition for its individual energy, $E_{\mathrm{NS}}$, does not include the gravitational influence of the $\mathrm{BH}$. It turns out that this way of superposing the NS is closer to the case of a test body. Indeed, if we look at the binding energy by considering the NS as a test body, we find that $E_{b}=M_{\mathrm{NS}}\left(1-E_{\mathrm{NS}} / M_{\mathrm{NS}}\right)$, i. e. the usual definition for test masses, and we obtain the correct Newtonian expression.

We could modify our description by performing an additional coordinate change to the NS model, prior to the superposition. This would result in extra parameters, which may be fixed so that the deformations of the NS produced by this coordinate change will account for the effects caused by the presence of the BH. While a detailed study of such modifications is beyond the scope of this paper, it will be interesting to study their effect in future work.

\section{E. On the Equations of Motion for the NS}

In a completely general setup the equations of motion for the matter fields associated with the NS just follow from the local conservation of energy and momentum, i.e. $\nabla_{\mu} T^{\mu \nu}=0$, which leads to the equations of magneto-hydrodynamics. In this paper, however, we are advocating an approximate approach that avoids solving hydrodynamical equations by reducing the number of physical degrees of freedom to a finite number, the hydro without hydro approach. More precisely, the idea is, that we can approximate the matter description by a simplified model that involves a finite number of parameters, $\left\{\lambda^{I}\right\}$, so that the associated energy-momentum distribution is determined in terms only of these parameters and the trajectory of a certain center of mass, $z^{\mu}$, that is, $T^{\mu \nu}=T^{\mu \nu}\left[z^{\rho} ; \lambda^{I}\right]$. Then, in order to update the matter sources that enter in our BSSN evolution equations we merely need to evolve the parameters $\left\{\lambda^{I}\right\}$ (referred to in this work as the internal motion) and the components of the trajectory $z^{\mu}$ (the external motion). In practice, evolution of these parameters is obtained from ordinary differential equations. This is a direct consequence of going from an infinite number degrees of freedom, as described by partial differential equations, to a finite number, described by ordinary differential equations.

For the case of extreme-mass-ratio binaries, the structure of the small object may be neglected as a first approximation, so that we do not need to consider the interior motion. The external motion, in turn, is given by the solution of the geodesic equations in the numerically constructed spacetime. In order to obtain a more realistic description of BH-NS binaries with similar masses, it is important to take into account the internal motion or, in other words, we need to allow for deformations of the NS associated with tidal forces arising from the presence of the $\mathrm{BH}$. To that end, an interesting approach is to consider the so-called affine stellar model introduced by Lattimer and Schramm [37] and further developed by Carter and Luminet [38, 39] and generalized for curved spacetimes in 40]. As it stands now, however, the affine model is applicable only to slowly varying general relativistic spacetimes [40, 41, 42], where a stationary background can be identified. This is an assumption that cannot be adopted in our framework, since we are interested in situations that involve strong and dynamical gravitational fields. Therefore, the affine model should be generalized appropriately for dynamical spacetimes. A particularly interesting way of doing this is based on Dixon's work [43, 44, 45, 46] (see also [4]). The idea is to use the multipole moments associated with the energy-momentum tensor to construct equations of motion for the center of mass, i. e. $z^{\mu}$, and for the extra parameters that describe the NS internal motion. In any case, the affine model has been used successfully to study the tidal interaction and disruption of stars by supermassive BHs [38, 40, 47], isolated rotating stars [48], stars in binary systems [9] and, more recently, gravitational signals arising from tidal interactions [50]. Another semi-analytic approach is the ellipsoidal energy variational or Roche-Riemann model [51] and its relativistic generalization [52] which is formally equivalent to the hydrostatic limit of the affine model.

In this paper, our goal is to investigate the stability of the numerical relativistic evolutions under the presence of moving matter distributions. To that end, we adopt the simplest possible evolution for the NS, namely a fixed trajectory around the $\mathrm{BH}$.

\section{COMPUTATIONAL FRAMEWORK AND NUMERICAL COMPUTATIONS}

The numerical results presented in this work have been obtained with the 3D numerical relativity MAYA code which has been described in detail in [13, 14]. The code uses the CACTUS computational toolkit 15] for parallelization, data input/output and horizon finding. Inside the CACTUS environment mesh refinement is provided by the CARPET 
TABLE I: Parameters and resulting mass and density for the six stellar models discussed in this work.

\begin{tabular}{c|ccc|ccc}
\hline \hline Model & $k$ & $n$ & $R_{\mathrm{NS}}\left[M_{\mathrm{BH}}\right]$ & $M_{\mathrm{NS}}\left[M_{\mathrm{BH}}\right]$ & $M_{\mathrm{NS}} / R_{\mathrm{NS}}$ & $\rho_{\mathrm{c}}\left[M_{\mathrm{BH}}^{-2}\right]$ \\
\hline 1 & 0.831 & 1 & 1 & $10^{-1}$ & $10^{-1}$ & $1.910^{-2}$ \\
2 & 0.652 & 1 & 1 & $10^{-2}$ & $10^{-2}$ & $10^{-3}$ \\
3 & 0.638 & 1 & 1 & $10^{-3}$ & $10^{-3}$ & 7.9 \\
4 & 0.00831 & 1 & 0.1 & $10^{-4}$ & $10^{-1}$ & $10^{-2}$ \\
5 & 0.00652 & 1 & 0.1 & $10^{-4}$ & $10^{-3}$ \\
6 & 0.00638 & 1 & 0.1 & & $7.910^{-2}$ \\
\hline \hline
\end{tabular}

package [16]. With regard to earlier versions of the MAYA code used for BH simulations, the evolution of extrememass-ratio binaries in the framework of the geodesic approximation method has made necessary some additions and modifications to the code. Foremost, these are the implementation of dynamic (moving) mesh-refinement and the addition of the matter source terms on the right hand sides of the Einstein field equations. Both will be described in more detail as we discuss the numerical simulations performed in this work.

\section{A. Description of the Numerical Implementation}

We first address the calculation of the TOV profiles. The TOV equations (37)-(39) constitute a boundary value problem for the variables $A(r), m(r)$ and $\rho(r)$. This system of equations is closed by an equation of state $p=p(\rho)$, chosen to be of polytropic type in this work. The boundary conditions are given by $m=0$ at the center as well as $p=0$ and $A=\sqrt{1-2 M_{\mathrm{NS}} / R_{\mathrm{NS}}}$ at the stellar surface. We solve the resulting system of ordinary differential equations using a standard relaxation scheme [53]. In practice, we calculate the TOV profiles during the initialization phase of the code and store them as functions of the radial variable $r$. The values required on the three-dimensional Cartesian grid are then obtained from third order polynomial interpolation.

The discretization of the BSSN evolution equations (23 28) has been implemented using centered second order stencils for spatial derivatives, except for the advection terms of type $\beta^{k} \partial_{k}$, for which second order accurate upwinding operators have been used. The integration in time is done using an iterative Crank-Nicholson scheme. As in simulations of vacuum spacetimes we have treated the spacetime singularities associated with the BHs by excising an area of finite size inside the apparent horizons (see [13, 14] for the details of the implementation of this technique).

\section{B. Analysis of the Initial Data}

In order to study the properties of the initial data proposed in this paper we have considered six different TOV models for the NS. The physical features of these models (the polytropic coefficient $k$, the polytropic index $n$, stellar radius $R_{\mathrm{NS}}$ and mass $M_{\mathrm{NS}}$, compactness ratio $M_{\mathrm{NS}} / R_{\mathrm{NS}}$, and central density $\rho_{\mathrm{c}}$ ) are shown in Table $\llbracket$ Notice, that the compactness ratios are small and hence some of the models would describe stars much less compact than astrophysical NSs.

From the values in Table \we find that the central density does not only increase with the compactness of the stellar model, but also when we make the star smaller, keeping constant the compactness ratio (see, for instance, models 1 and 4 ). This is an expected result: if we reduce the mass $M_{\mathrm{NS}}$ while keeping the compactness ratio $M_{\mathrm{NS}} / R_{\mathrm{NS}}$ constant, the density behaves like

$$
\rho \sim \frac{M_{\mathrm{NS}}}{R_{\mathrm{NS}}^{3}}=\frac{1}{M_{\mathrm{NS}}^{2}} \frac{M_{\mathrm{NS}}^{3}}{R_{\mathrm{NS}}^{3}}=\frac{\text { const }}{M_{\mathrm{NS}}^{2}} .
$$

That is, the density scales as the inverse of the square of the mass. The values in Table \ confirm this relation. From the computational point of view, this feature represents a considerable challenge. For more extreme mass ratios ( smaller $M_{\mathrm{NS}}$ ) we do not only need to decrease the grid spacing linearly with the size of the star. Further increase in resolution is necessary to adequately resolve the steep gradients resulting from the increasingly larger matter densities and curvature encountered for smaller values of $M_{\mathrm{NS}}$. Even with the availability of mesh refinement, as discussed below, the computational demands for simulating low mass compact sources quickly become prohibitively costly. It is for this reason that we also consider the less compact models listed in Table \. We emphasize, however, that all models under consideration here are orders of magnitude more compact than those used in similar studies in the 
TABLE II: Structure of the refinement levels as used in the study of the initial data. The two values for the grid spacing correspond to the coarse and fine resolution in the convergence analysis. The boundaries in the $z$-direction are $z_{\text {min }}=0$ and $z_{\max }=y_{\max }$ on all levels and have not been listed explicitly. Levels 7-9 are only used for stellar models 4-6.

\begin{tabular}{|c|c|c|c|c|c|c|c|c|c|c|}
\hline \multirow{2}{*}{$\begin{array}{c}\text { Refinement } \\
\text { Level }\end{array}$} & \multirow{2}{*}{$h_{1}\left[M_{\mathrm{BH}}\right]$} & \multirow[b]{2}{*}{$h_{2}\left[M_{\mathrm{BH}}\right]$} & \multicolumn{4}{|c|}{ Grids centered around the $\mathrm{BH}$} & \multicolumn{4}{|c|}{ Grids centered around the NS } \\
\hline & & & $x_{\min }\left[M_{\mathrm{BH}}\right]$ & $x_{\max }\left[M_{\mathrm{BH}}\right]$ & $y_{\min }\left[M_{\mathrm{BH}}\right]$ & $y_{\max }\left[M_{\mathrm{BH}}\right]$ & $x_{\min }\left[M_{\mathrm{BH}}\right]$ & $x_{\max }\left[M_{\mathrm{BH}}\right]$ & $y_{\min }\left[M_{\mathrm{BH}}\right]$ & $y_{\max }\left[M_{\mathrm{BH}}\right]$ \\
\hline 1 & 2.88 & 1.92 & -103.68 & 103.68 & -103.68 & 103.68 & & & & \\
\hline 2 & 1.44 & 0.96 & -51.84 & 51.84 & -51.84 & 51.84 & & & & \\
\hline 3 & 0.72 & 0.48 & -25.92 & 25.92 & -25.92 & 25.92 & & & & \\
\hline 4 & 0.36 & 0.24 & -12.96 & 12.96 & -12.96 & 12.96 & & & & \\
\hline 5 & 0.18 & 0.12 & -4.32 & 4.32 & -4.32 & 4.32 & 6.48 & 10.80 & -2.16 & 2.16 \\
\hline 6 & 0.09 & 0.06 & & & & & 7.56 & 9.72 & -1.08 & 1.08 \\
\hline 7 & 0.045 & 0.03 & & & & & 8.1 & 9.18 & -0.54 & 0.54 \\
\hline 8 & 0.0225 & 0.015 & & & & & 8.37 & 8.91 & 0.27 & 0.27 \\
\hline 9 & 0.01125 & 0.0075 & & & & & 8.505 & 8.775 & 0.135 & 0.135 \\
\hline
\end{tabular}

literature 21]. Below we will discuss the numerical demands arising from high compactness more quantitatively, when we discuss the constraint violations of the superposed data. First, we test the numerical implementation of the boosted TOV solution by studying the convergence of the constraints in the absence of a $\mathrm{BH}$.

The initial data constructed according to the procedure described above results in exact solutions of Einstein's equations in various limits. In particular, by setting the mass of the BH to zero we recover the solution of a boosted TOV star in KS-like coordinates. We emphasize, that, in the absence of the BH, there is no fundamental length scale $M_{\mathrm{BH}}$, so that the numerical values obtained for the NS no longer have the unambiguous meaning as before. A key motivation for this study, however, is to probe the suitability of the numerical framework used for the simulations. For this purpose we construct the numerical grid using the same numerical values as if a $\mathrm{BH}$ of mass $M_{\mathrm{BH}}$ were present. In this work we limit our discussion to non-spinning BHs and stellar models. In consequence, the configurations under study are inherently symmetric about the orbital plane. It is sufficient, for this reason, to evolve data only in the bitant $z>0$ and impose symmetry conditions in the orbital $x, y$ plane.

In order to achieve maximum resolution at moderate computational cost, we use Fixed Mesh Refinement for all simulations in this work. Inside the MAYA code, Mesh Refinement is implemented via the CARPET package [16, 17]. In contrast to the static fixed mesh refinement used for the head-on collisions in [24] the type of scenario investigated here will eventually require dynamic mesh refinement to accommodate the orbital motion of the stellar object around the BH. We return to this issue later, when we will discuss time evolutions. For the initial data under consideration here, we restrict our attention to the initial setup of the refinement levels. The initial position of the star in this case is $x=8.64, y=z=0$ and it has an initial boost velocity given by $v_{x}=v_{z}=0, v_{y}=0.34$. For the stellar models 1-3 of Table Wwe use a set of 5 nested boxes around the BH plus a set of two nested refinement components centered around the NS. For models 4-6 we add three further refinement levels centered on the NS. The exact specifications of the refinement levels are given in Table III

For the convergence analysis we have calculated the data for two different grid spacings $h_{1}$ and $h_{2}=h_{1} / 1.5$ as listed in the table. Because an isolated boosted TOV star is a solution of the Einstein equations we expect the Hamiltonian and momentum constraints, given by equations (1314), to converge to zero at second order. Numerically this implies that the constraint violations obtained for the high resolution calculation should be $1.5^{2}$ smaller than those obtained at coarse resolution.

Figure 1 demonstrates second order convergence for the Hamiltonian and the norm $\|\mathcal{M}\|=\sqrt{\sum_{i} \mathcal{M}_{i}^{2}}$ of the momentum constraints along the $x$-axis, obtained for the most compact model of Table $\amalg$ model 4 . We find similar results for all constraint functions on arbitrary axes. A similar analysis at coarser resolution $\left(1.5 h_{1}\right.$ instead of $\left.h_{2}\right)$, however, gave less satisfactory results, indicating that the resolutions used here are necessary for model 4 . For the other, less extreme, models we find correspondingly less strict requirements on the resolution to achieve second order convergence.

We now turn our attention to the binary data obtained for non-vanishing mass of the BH. In the continuum limit the resulting data are a solution of the Einstein equations only as the separation of the two objects becomes infinite. We therefore do not expect the constraint violations to converge to zero. On the other hand, the constraints will never be satisfied exactly in a numerical simulation because of the finite numerical accuracy. In order to assess the significance of the constraint violations inherent to the superposed data relative to the limitations in numerical accuracy we follow the same approach as in [14] and study the constraint violations at different resolutions. If the constraint violations decrease at about second order for higher resolutions we conclude the inherent constraint violations (due to the fact 

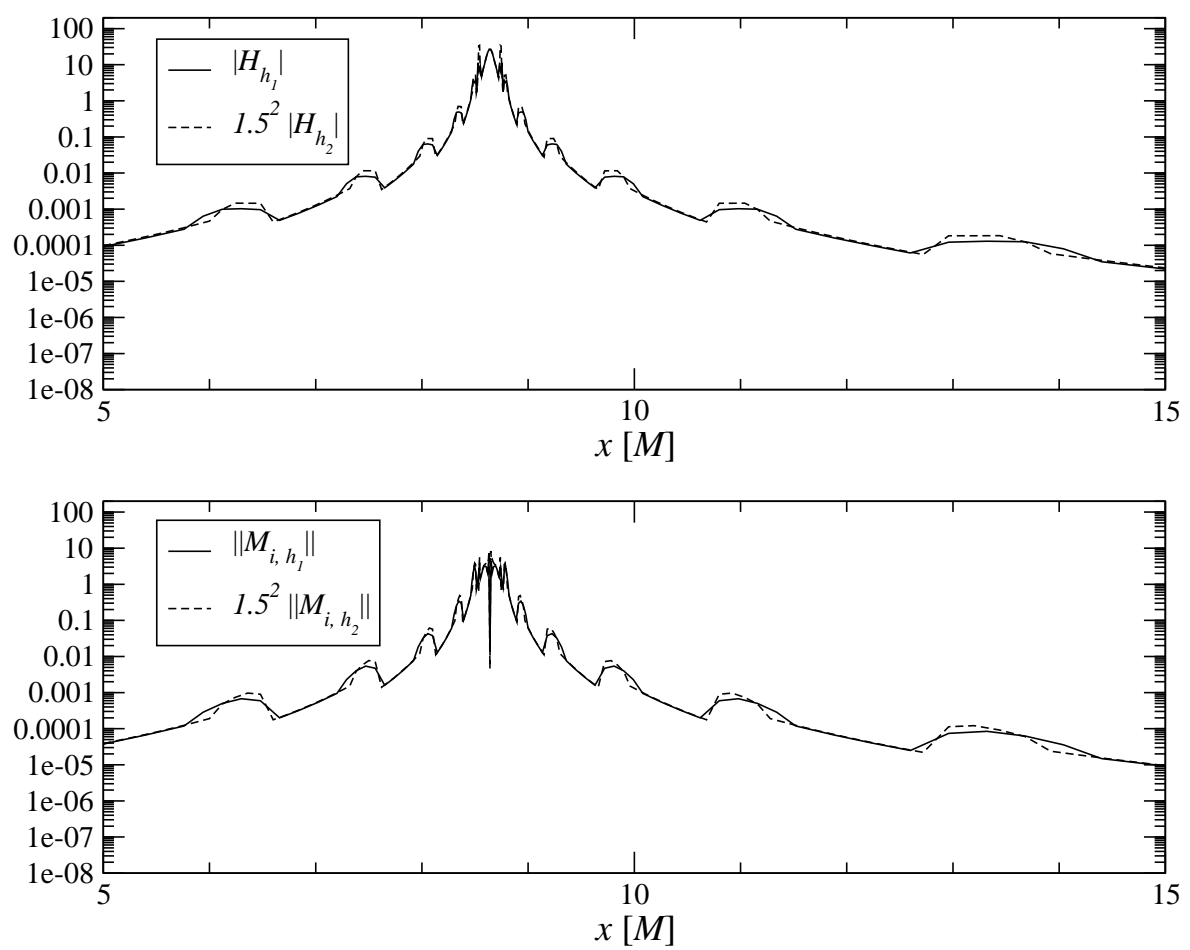

FIG. 1: Constraint violation of the Hamiltonian and the norm of the momentum constraint along the $x$-axis for stellar model 4 in the absence of a BH. The constraints obtained at high resolution have been amplified by $1.5^{2}$.

that we are not solving them) to be insignificant relative to the purely numerical error.

For this purpose we show in Figure 2 the convergence of the Hamiltonian constraint and the norm $\|\mathcal{M}\|$ of the momentum constraints along the $x$-axis. For clarity we have used a logarithmic scale for the distance from the origin in both directions. As in the case of an isolated star, we find that the constraints converge away at second order for the resolutions used here. The only exception is the region of the star, where the non-linear interaction between the gravitational fields of the two objects is strongest and we expect the constraint violations inherent to this data construction to be largest. It is these constraint violations which represent the approximative nature of our approach to study this type of binary configurations.

Figure 2 also illustrates the high computational demands for simulations of more compact objects. The two models (3 and 4 of Table \ used in the figure represent the least and most compact stars. Clearly the constraint violations found for model 4 in the upper two panels are very large compared to the average violations near the BH. This is an artifact of the high densities and curvature encountered near the small star in combination with resolutions currently available with our computers. In contrast the constraint violations found for model 3 in the lower panels is substantially smaller than those observed near the BH. We emphasize that this issue is separate from the convergence properties mentioned above. In fact, the convergence properties found for model 4 are closer to second order than those found for model 3 . We attribute this to the fact that the constraint violations near the very compact object 4 are dominated by the discretization errors arising from the insufficient resolution near the stellar center. For these reasons we study in the next subsection evolutions of the less compact model 3 only, for which we are confident to achieve sufficient resolution, even inside the star.

\section{Time evolutions}

In this section we present time evolutions of the initial data presented above. Following our previous discussions, we focus on the implementation of the different elements of this type of simulations in the framework of moving refinement components with emphasis on the stability of the evolutions. 

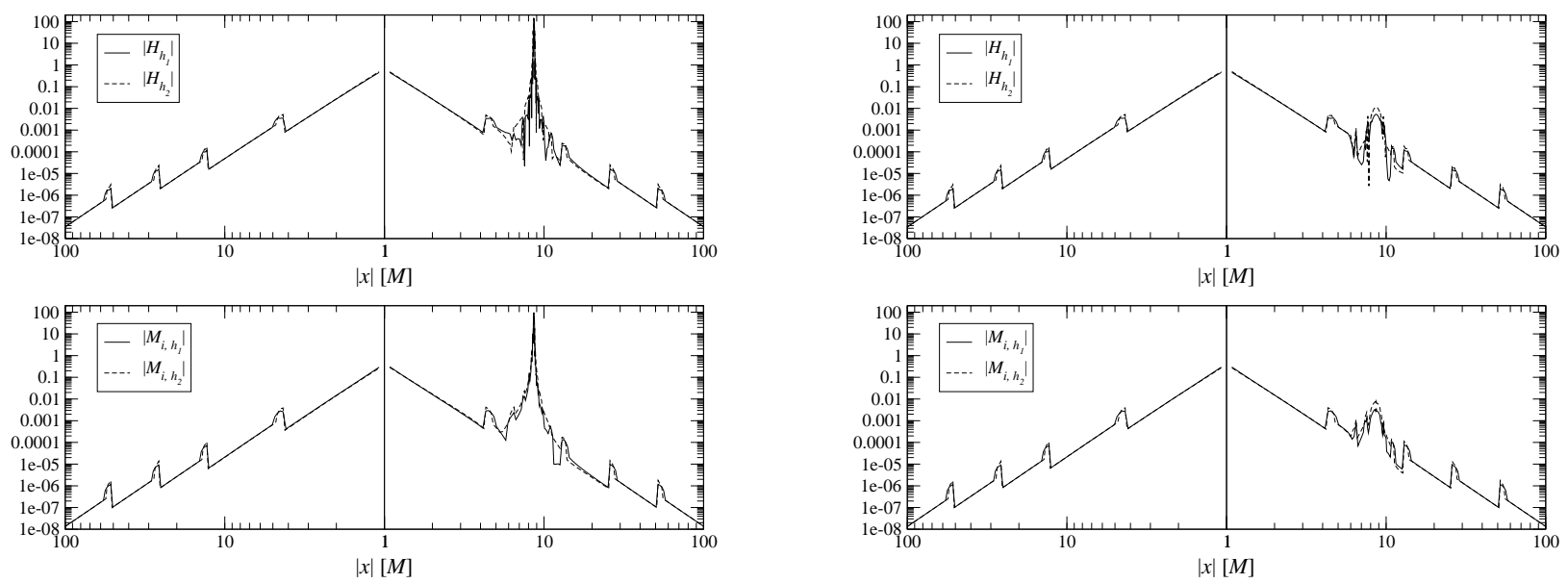

FIG. 2: Constraint violation of the Hamiltonian and momentum constraints along the $x$-axis for stellar model 4 (left panels) and 3 (right panels). The constraints obtained at high resolution have been amplified by $1.5^{2}$.

TABLE III: Structure of the refinement levels as used for the time evolution.

\begin{tabular}{|c|c|c|c|c|c|}
\hline Refinement Level & $h\left[M_{\mathrm{BH}}\right]$ & $x_{\min }\left[M_{\mathrm{BH}}\right]$ & $x_{\max }\left[M_{\mathrm{BH}}\right]$ & $y_{\min }\left[M_{\mathrm{BH}}\right]$ & $y_{\max }\left[M_{\mathrm{BH}}\right]$ \\
\hline \begin{tabular}{l|}
1 \\
\end{tabular} & 4.00 & -144.00 & 144.00 & -144.00 & 144.00 \\
\hline 2 & 2.00 & -72.00 & 72.00 & -72.00 & 72.00 \\
\hline 3 & 1.00 & -36.00 & 36.00 & -36.00 & 36.00 \\
\hline 4 & 0.50 & -18.00 & 18.00 & -18.00 & 18.00 \\
\hline 5 & 0.25 & -12.00 & 12.00 & -12.00 & 12.00 \\
\hline 6 & 0.125 & 5.00 & 9.00 & -2.00 & 2.00 \\
\hline
\end{tabular}

To this end, we have evolved the superposed data of a $\mathrm{BH}$ of mass $M_{\mathrm{BH}}=1$ and stellar model 3 as listed in Table Because of memory restrictions of the available computational resources we have evolved this binary system using a distance $d=7 \mathrm{M}$ and a grid setup slightly different from that of Table The grid specifications used in the evolution are listed in Table III Of the refinement levels listed there, number 1 to 5 remain fixed throughout the evolution. Level 6, however, is required to follow the motion of the stellar object and thus needs to move. For this purpose we use the regridding operation implemented inside CARPET. This operation allows for interpolation of function values from coarser onto finer refinement levels. This operation is regularly performed in mesh refinement simulations of Berger-Oliger type to provide boundary conditions for the inner levels, the so-called prolongation. In case of a moving high resolution grid component, the same operation is used to fill the new points on that component with valid data from the coarser levels. Inside the MAYA code we use this feature by adjusting the specifications, i.e. $x_{\min }, x_{\max }, y_{\min }$ and $y_{\max }$, of refinement level 6 in the course of the evolution.

We have implemented two alternative ways of controlling these values. The first uses the tracking of the $\mathrm{BH}$ singularity, as provided, for example, by an apparent horizon finder, and moves the center of the refinement component by a corresponding amount. Because this method is inherently restricted to BHs, we also allow for the center of the component to follow a user specified trajectory. This second method is realized for the case of the stellar object discussed in this work. The trajectory is the same as that used for the motion of the stellar object itself. In Fig. 3. we show four snapshots of the ensuing evolution. The figure shows the trace of the extrinsic curvature obtained at times $t=0,30 \mathrm{M}, 70 \mathrm{M}$ and $120 \mathrm{M}$. While the $\mathrm{BH}$ is represented by the large central throat, the stellar object manifests itself in the form of the small perturbation initially visible to the right of the $\mathrm{BH}$ (upper left panel).

The simulations we have carried out last at least for an evolution time of $167 M$, corresponding to about one and a half orbits, without showing signs of instability. Furthermore, no elaborate fine-tuning of the parameters was necessary to achieve evolutions. In conclusion, simulations on orbital timescales appear to be achievable rather straightforwardly using the approach discussed in this work. 

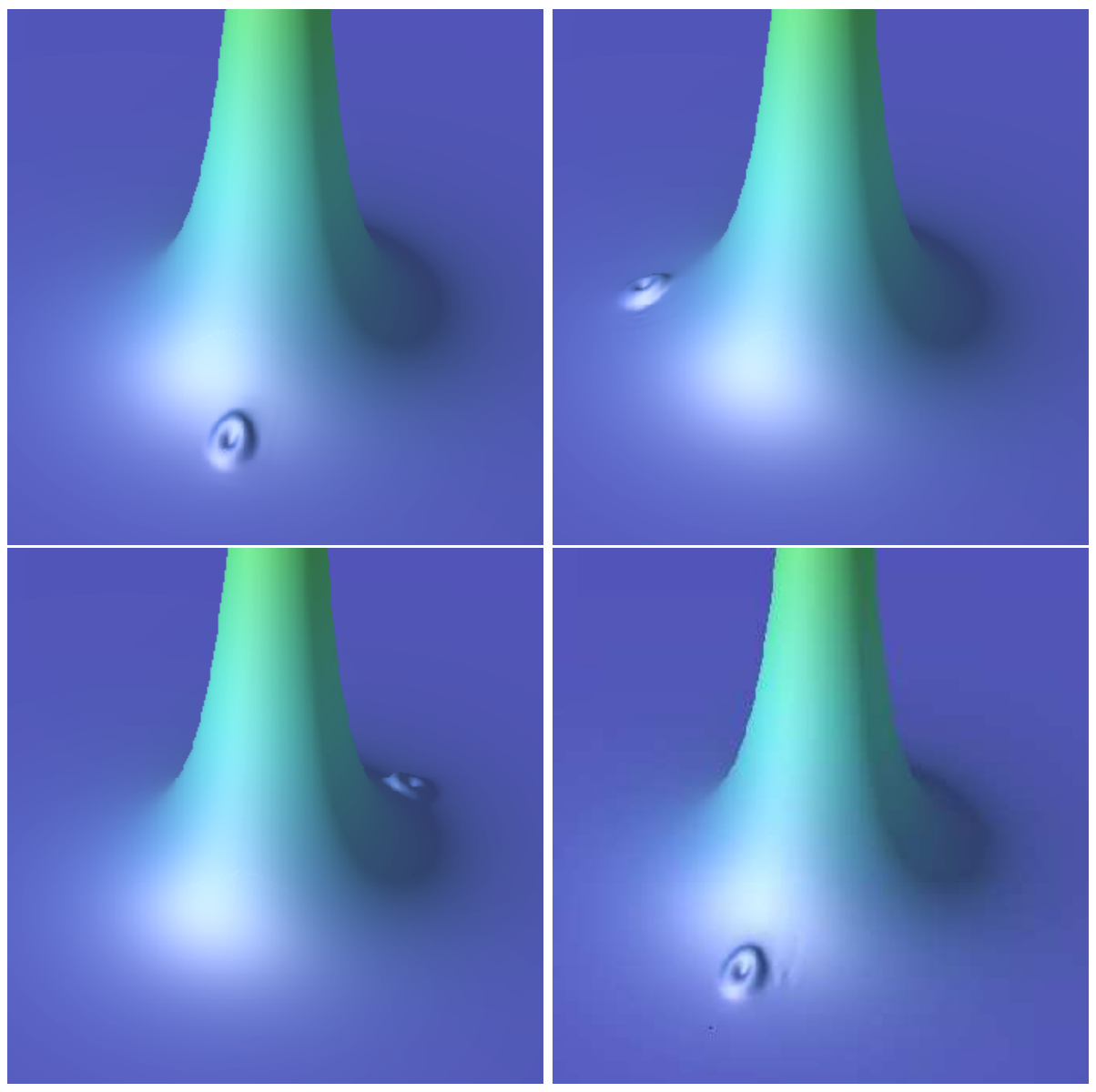

FIG. 3: Snapshots of the time evolution of a NS model type model 3 orbiting a massive BH with orbital frequency $\omega=$ $0.054 M_{\mathrm{BH}}^{-1}$.

\section{CONCLUSIONS}

In this paper we have introduced a framework to study BH-NS binaries using a fully non-linear evolution of the spacetime geometry and an approximate evolution of the NS matter sources based on the reduction of the degrees of freedom of the NS, so that hydrodynamical calculations are avoided. The remaining degrees of freedom, including the motion of the NS are governed by ODEs. We expect this framework to be useful to investigate certain dynamical regimes of the BH-NS binary system relevant for the point of view of gravitational-wave astronomy.

The construction of the initial data is performed by generalizing the superposition technique of [20], as applied to $\mathrm{BH}$ binaries in KS form, to BH-NS binaries in KS-like coordinates. In order to make this approach functional in numerical relativity using three-dimensional Cartesian coordinates, we found it necessary to modify the standard coordinate transformation to KS coordinates inside the NS. In particular, we used a coordinate transformation that avoids the appearance of a coordinate singularity at the stellar centre, while preserving the light-like geodesic structure of the coordinates outside the star.

We have studied the properties of the initial data thus obtained for a set of stellar models with different values of the compactness and mass ratios. An adequate description of extreme-mass-ratio binaries involving compact stars is only made possible by the use of advanced mesh-refinement techniques. We have demonstrated second-order convergence of the Hamiltonian and momentum constraints in the case of an isolated NS (corresponding to an infinite BH-NS separation). For finite separations we still observe second-order convergence of the constraints over large parts of the computational domain. We thus conclude that these initial data sets are as suitable for the description of BH-NS binaries as the pure $\mathrm{BH}-\mathrm{BH}$ analog previously discussed in the literature.

Finally, we have investigated the feasibility of evolving such scenarios in time using modern methods of numerical relativity. In the numerical simulations considered in this work for testing purposes, we have restricted ourselves to the simple case in which the NS follows a prescribed trajectory. Foremost, our interest was focused on the stability of the 
resulting simulations and the demands on computational resources. We have found that for sufficiently extreme mass ratios, long-term stable simulations on orbital time scales can be achieved straightforwardly using standard evolution schemes for solving the Einstein field equations. As the equal-mass limit is approached, the stability properties deteriorate because of the strong dynamics in the vicinity of the $\mathrm{BH}$. In this limit, our approach no longer provides an adequate description of the matter sources, however. The scenarios of immediate interest for our approximation technique are thus within the range of capability of our evolution code.

With regard to the compactness of the NS we find that the combination of extreme mass ratios with high compactness of the NS result in very steep gradients. These, in turn, demand a very high resolution and a large number of refinement levels. Currently we do not have the computational resources available to provide such resolution in long-term evolutions. We have therefore studied the time evolutions of less compact objects, but still orders of magnitude more compact than what has hitherto been considered using this type of approach. Such studies are well within the scope of current resources and can be refined in the future, by adding radiation-reaction effects to the dynamics and/or internal degrees of freedom of the NS to study different aspects of these systems in the context of gravitational-wave astronomy.

Acknowledgements: The authors acknowledge the support of the Center for Gravitational Wave Physics funded by the National Science Foundation under Cooperative Agreement PHY-0114375, and support from NSF grant PHY0244788 to Penn State University. The authors wish to thank Erik Schnetter for help with the implementation of moving refinement boxes. U.S. acknowledges support from the SFB/TR 7 "Gravitational Wave Astronomy" of the German Science Foundation and the hospitality of the Albert Einstein Institute (Potsdam) during a visit.

[1] K. Taniguchi, T. W. Baumgarte, J. A. Faber, and S. L. Shapiro, Phys. Rev. D72, 044008 (2005), astro-ph/0505450.

[2] J. A. Faber, T. W. Baumgarte, S. L. Shapiro, K. Taniguchi, and F. A. Rasio, Phys. Rev. D73, 024012 (2006), astro$\mathrm{ph} / 0511366$.

[3] J. A. Faber, T. W. Baumgarte, S. L. Shapiro, and K. Taniguchi (2006), astro-ph/0603277.

[4] N. Gehrels, C. L. Sarazin, P. T. O'Brien, B. Zhang, L. Barbier, S. D. Barthelmy, A. Blustin, D. N. Burrows, J. Cannizzo, J. R. Cummings, M. Goad, S. T. Holland, et al., Nature 437, 851 (2005).

[5] J. S. Villasenor, D. Q. Lamb, G. R. Ricker, J.-L. Atteia, N. Kawai, N. Butler, Y. Nakagawa, J. G. Jernigan, M. Boer, G. B. Crew, T. Q. Donaghy, J. Doty, et al., Nature 437, 855 (2005).

[6] D. B. Fox, D. A. Frail, P. A. Price, S. R. Kulkarni, E. Berger, T. Piran, A. M. Soderberg, S. B. Cenko, P. B. Cameron, A. Gal-Yam, M. M. Kasliwal, D.-S. Moon, et al., Nature 437, 845 (2005).

[7] J. Hjorth, D. Watson, J. P. U. Fynbo, P. A. Price, B. L. Jensen, U. G. Jørgensen, D. Kubas, J. Gorosabel, P. Jakobsson, J. Sollerman, K. Pedersen, and C. Kouveliotou, Nature 437, 859 (2005).

[8] E. Berger, P. A. Price, S. B. Cenko, A. Gal-Yam, A. M. Soderberg, M. Kasliwal, D. C. Leonard, P. B. Cameron, D. A. Frail, S. R. Kulkarni, D. C. Murphy, W. Krzeminski, et al., Nature 438, 988 (2005).

[9] N. R. Tanvir, R. Chapman, A. J. Levan, and R. S. Priddey, Nature 438, 991 (2005).

[10] S. D. Barthelmy, G. Chincarini, D. N. Burrows, N. Gehrels, S. Covino, A. Moretti, P. Romano, P. T. O’Brien, C. L. Sarazin, C. Kouveliotou, M. Goad, S. Vaughan, et al., Nature 438, 994 (2005).

[11] T. W. Baumgarte and S. L. Shapiro, Phys. Rev. D59, 024007 (1999), gr-qc/9810065.

[12] M. Shibata and T. Nakamura, Phys. Rev. D52, 5428 (1995).

[13] D. Shoemaker, K. Smith, U. Sperhake, P. Laguna, E. Schnetter, and D. Fiske, Class. Quantum Grav. 20, 3729 (2003), gr-qc/0301111.

[14] U. Sperhake, K. L. Smith, B. Kelly, P. Laguna, and D. Shoemaker, Phys. Rev. D 69, 024012 (2004), gr-qc/0307015.

[15] CACTUS, www. cactuscode.org.

[16] CARPET, www. carpetcode.org.

[17] E. Schnetter, S. H. Hawley, and I. Hawke, Class. Quant. Grav. 21, 1465 (2004), gr-qc/0310042.

[18] R. C. Tolman, Phys. Rev. 55, 364 (1939).

[19] J. R. Oppenheimer and G. M. Volkoff, Phys. Rev. 55, 374 (1939).

[20] R. A. Matzner, M. F. Huq, and D. Shoemaker, Phys. Rev. D59, 024015 (1999), gr-qc/9805023.

[21] N. T. Bishop, R. Gomez, S. Husa, L. Lehner, and J. Winicour, Phys. Rev. D68, 084015 (2003), gr-qc/0301060.

[22] P. Marronetti et al., Phys. Rev. D62, 024017 (2000), gr-qc/0001077.

[23] E. Bonning, P. Marronetti, D. Neilsen, and R. Matzner, Phys. Rev. D68, 044019 (2003), gr-qc/0305071.

[24] U. Sperhake, B. Kelly, P. Laguna, K. L. Smith, and E. Schnetter, Phys. Rev. D71, 124042 (2005), gr-qc/0503071.

[25] R. Arnowitt, S. Deser, and C. W. Misner, in Gravitation: An introduction to current research, edited by L. Witten (Wiley, New York, 1962), pp. 227-265.

[26] R. M. Wald, General Relativity (The University of Chicago Press, Chicago, 1984).

[27] J. York, Jr., in Sources of gravitational radiation, edited by L. Smarr (Cambridge University Press, Cambridge, 1979), p. 83.

[28] H.-J. Yo, T. W. Baumgarte, and S. L. Shapiro, Phys. Rev. D 66, 084026 (2002), gr-qc/0209066.

[29] A. Lichnerowicz, Théories Relativistes de la Gravitation et de l'Electromagnétisme (Masson, Paris, 1955). 
[30] A. Lichnerowicz, C. R. Acad. Sci. 273, 528 (1971).

[31] B. J. Kelly, The Next Generation of Binary Black Hole Head-on Collisions, and their Aftermath, Ph.D. thesis, The Pennsylvania State University (2004).

[32] A. Lichnerowicz, J. Math. Pures et Appl. 23, 3763 (1944).

[33] J. York, Jr., Phys. Rev. Lett. 26, 1656 (1971).

[34] J. York, Jr., Phys. Rev. Lett. 28, 1082 (1972).

[35] J. York, Jr., J. Math. Phys. 14, 456 (1973).

[36] G. B. Cook, Living Rev. Rel. 3, 5 (2000), gr-qc/0007085.

[37] J. M. Lattimer and D. N. Schramm, ApJ 210, 549 (1976).

[38] B. Carter and J.-P. Luminet, $A \xi A$ A 121, 97 (1983).

[39] B. Carter and J. P. Luminet, MNRAS 212, 23 (1985).

[40] J.-P. Luminet and J.-A. Marck, MNRAS 212, 57 (1985).

[41] B. Mashhoon, ApJ 197, 705 (1975).

[42] B. Mashhoon, ApJ 216, 591 (1977).

[43] W. G. Dixon, Royal Society of London Proceedings Series A 314, 499 (1970).

[44] W. G. Dixon, Royal Society of London Proceedings Series A 319, 509 (1970).

[45] W. G. Dixon, Royal Society of London Philosophical Transactions Series A 277, 59 (1974).

[46] W. G. Dixon, General Relativity and Gravitation 8, 595 (1977).

[47] P. Diener, A. G. Kosovichev, E. V. Kotok, I. D. Novikov, and C. J. Pethick, MNRAS 275, 498 (1995).

[48] D. Lai, F. A. Rasio, and S. L. Shapiro, ApJ 437, 742 (1994).

[49] D. Lai and S. L. Shapiro, ApJ 443, 705 (1995).

[50] C. Casalvieri, V. Ferrari, and A. Stavridis (2005), astro-ph/0508190.

[51] D. Lai, F. A. Rasio, and S. L. Shapiro, ApJS 88, 205 (1993).

[52] P. Wiggins and D. Lai, ApJ 532, 530 (2000).

[53] W. H. Press, B. P. Flannery, S. A. Teukolsky, and W. T. Vetterling, Numerical Recipes: The Art of Scientific Computing (Cambridge University Press, Cambridge (UK) and New York, 1992). 